\title{
Type 1 diabetes mellitus and hyperadrenocorticism in a ferret
}

\author{
A. Boari • V. Papa • F. Di Silverio • G. Aste • D. Olivero • \\ F. Rocconi
}

Published online: 7 May 2010

(C) Springer Science+Business Media B.V. 2010

\begin{abstract}
Diabetes mellitus (DM) was diagnosed in a 6-year-old neutered male ferret with polyuria/ polydipsia, symmetrical alopecia, and weight loss. Laboratory tests revealed severe hyperglycemia, glucosuria, and increased steroid hormone profile. Abdominal ultrasound revealed a bilateral enlargement of the adrenal glands. Significant clinical improvement was achieved with insulin- and leuprolide acetate-based therapy. After 2 months of therapy, the ferret showed a severe ketoacidosis, and the owner decided to euthanize the animal. Histological findings revealed carcinoma of the left adrenal cortex and cortical hyperplasia of the right adrenal gland. Moderate, chronic, and active pancreatitis with a marked decrease in the number of $\beta$-cells was also present. This is the first reported case of type 1 DM associated with hyperadrenocorticism and chronic pancreatitis in a ferret.
\end{abstract}

Keywords Ferret $\cdot$ Diabetes mellitus $\cdot$ Pancreatitis $\cdot$ Hyperadrenocorticism

\author{
Abbreviations \\ DM diabetes mellitus \\ HAC hyperadrenocorticism \\ DKA diabetic ketoacidosis \\ PBGM portable blood glucose meter
}

\section{Introduction}

Spontaneous DM is very uncommon in ferrets and is not well documented in the veterinary literature (Chen 2008). The clinical signs of diabetes in ferrets are similar to those of other species and are mainly based on polyphagia, weight loss, polyuria, and polydipsia. Diabetic

\footnotetext{
A. Boari $(\bowtie) \cdot$ V. Papa $\cdot$ F. Di Silverio $\cdot$ G. Aste $\cdot$ F. Rocconi

Dipartimento di Scienze Cliniche Veterinarie, Università degli Studi di Teramo, viale F. Crispi 212, 64100 Teramo, Italy

e-mail: aboari@unite.it

D. Olivero

BIEsseaA Laboratorio Analisi Veterinarie, Milano, Italy
} 
ferrets may appear lethargic and anorexic, in particular when DKA is present (Carpenter and Novilla 1977; Benoit-Biancamano et al. 2005). A diagnosis of DM is based on compatible clinical signs, a persistent blood glucose concentration more than $400 \mathrm{mg} / \mathrm{dL}$, and glucosuria (Quesenberry and Rosenthal 2003). A complete blood count, serum biochemistry, and radiography and ultrasonography are recommended to evaluate any concurrent condition. Since there are few reports of spontaneous DM in ferrets, it has not yet been determined what DM type affects this species. In the two cases of spontaneous DM where histological evaluation of the pancreas was performed, histological stains revealed an adequate number of $\beta$-granules in the pancreatic islets. This aspect suggests that $\mathrm{DM}$ is derived from either inadequate release of insulin or peripheral resistance to insulin (Carpenter and Novilla 1977; Benoit-Biancamano et al. 2005). Spontaneous HAC is a common disorder that results in an excessive production of estrogens, androgens, and estrogen-related compounds by the adrenal cortex (Quesenberry and Rosenthal 2003). The present paper aims to describe the first case of type $1 \mathrm{DM}$ in a ferret with concomitant pancreatitis and HAC.

\section{Case report}

A 6-year-old neutered male ferret (Mustela putorius furo), weighing $1,100 \mathrm{~kg}$, was admitted to the Department of Veterinary Clinical Sciences, University of Teramo, with a history of 5 weeks of polyphagia, polyuria, polydipsia, and weight loss. During the physical examination, the ferret presented moderate emaciation, mild muscle atrophy, moderate depression, and a bilateral symmetrical non-itchy alopecia. An immediate determination of blood glucose and ketone concentrations, using a PBGM (MediSense ${ }^{\circledR}$ Optium, Abbott Laboratories, UK), revealed hyperglycemia (398 mg/dL; reference range: 94-207 mg/dL) and low blood ketone levels $(0.1 \mathrm{mmol} / \mathrm{L})$. Initial diagnostic tests included a complete blood cell count, biochemical analysis, and urinalysis. Abnormal serum biochemical results confirmed hyperglycemia $(431 \mathrm{mg} / \mathrm{dL})$, hypoalbuminemia $(2.8 \mathrm{~g} / \mathrm{dL}$; reference range: $3.3-4.1 \mathrm{~g} / \mathrm{dL})$, and increased concentrations of both cholesterol $(384 \mathrm{mg} / \mathrm{dL}$; reference range: 119-201 mg/ $\mathrm{dL}$ ) and serum urea nitrogen $(76 \mathrm{mg} / \mathrm{dL}$; reference range: $12-43 \mathrm{mg} / \mathrm{dL})$. Urinalysis revealed a urine specific gravity of 1.032, marked glucosuria, and absence of ketones. A bacteriological examination of the urine was negative. According to the clinical signs of persistent hyperglycemia as well as glucosuria, a diagnosis of DM was made. Altered echogenicity and echotexture of the pancreas and an enlargement of both adrenal glands with a rounded appearance and heterogeneous structure became evident during the abdominal ultrasonography. The steroid hormone profile determined by the Clinical Endocrinology Service, University of Tennessee, showed an increased concentration of estradiol $331 \mathrm{pmol} / \mathrm{L}$ (reference range: $30-180 \mathrm{pmol} / \mathrm{L}$ ), $17 \alpha$-hydroxy-progesterone $5.5 \mathrm{nmol} / \mathrm{L}$ (reference range: $0-0.80 \mathrm{nmol} / \mathrm{L}$ ), and androstenedione $17.0 \mathrm{nmol} / \mathrm{L}$ (reference range: $0-15 \mathrm{nmol} / \mathrm{L}$ ).

Based on these data, a diagnosis of concomitant HAC was made. Normalization of blood glucose concentrations was attempted by subcutaneous administration of 0.5 IU porcine lente insulin (Caninsulin ${ }^{\circledR}$, Intervet) every $12 \mathrm{~h}$. The ferret was fed a commercial diet (m/d; Hill's) divided into four small meals. Furthermore, intramuscular administration of $100 \mu \mathrm{g}$ leuprolide acetate (Enantone ${ }^{\circledR}$, Takeda) was performed to stabilize the ferret before adrenal gland surgery. Data on water and food consumption, urine production, glucosuria, and ketonuria were obtained on a daily basis. Home monitoring of clinical signs and urinary glucosuria was initially unacceptable, and therefore the insulin dosage was increased to 
$1 \mathrm{IU}$ every $12 \mathrm{~h}$ after 7 days of insulin-based therapy. Such a dose led to a significant improvement in the ferret's clinical conditions. Subsequent examinations revealed good general conditions with an increase in body weight $(1,230 \mathrm{~kg})$. Urinalysis showed a low specific gravity (1.014) and glucosuria. At this point, the owner declined the surgery and a second dose of leuprolide acetate was injected intramuscularly.

During the next 3 weeks, the ferret revealed good clinical conditions and partial hair regrowth. Fifty days after its first admission, the ferret showed disorexia and the owner decided not to administrate insulin despite the presence of marked glucosuria and ketonuria. The ferret was readmitted due to severe depression, disorexia, polyuria, polydipsia, and weight loss. During the physical examination, severe lethargy, dehydration, bilateral chemosis, and hypothermia $\left(37.2^{\circ} \mathrm{C}\right)$ with weight loss to $800 \mathrm{~g}$ were present. Abnormal serum biochemical results included hyperglycemia $(265 \mathrm{mg} / \mathrm{dL})$, marked hyper ketonemia $(>6.0 \mathrm{mmol} / \mathrm{L})$, and increased serum urea nitrogen $(76 \mathrm{mg} / \mathrm{dL})$ and creatinine $(0.72 \mathrm{mg} / \mathrm{dL}$; reference range: $0.2-$ $0.6 \mathrm{mg} / \mathrm{dL}$ ). Severe metabolic acidosis and hypokalemia ( $\mathrm{pH} 7.052$; bicarbonate: $9.4 \mathrm{mmol} / \mathrm{L}$; $\mathrm{K}: 3.5 \mathrm{mmol} / \mathrm{L}$ ) were detected on venous blood gas analysis. Urinalysis revealed a low specific gravity (1.019), severe glucosuria, and moderate ketonuria. Few hypoechoic lesions within the pancreas appeared on abdominal ultrasonography. A diagnosis of DKA was made. The ferret was hospitalized and treatment consisted of fluids $(0.9 \%$ sodium chloride in a $5 \%$ glucose solution supplemented with $30 \mathrm{mEq} / \mathrm{L}$ potassium chloride), and regular insulin administration (0.1 IU at intermittent intramuscular injections every 4-6 h). Due to the severe metabolic acidosis, $0.4 \mathrm{mEq}$ sodium bicarbonate was administered. Blood glucose levels and blood gas parameters were monitored every 1 and $4 \mathrm{~h}$, respectively. A subcutaneous administration of $10 \mathrm{mg} / \mathrm{kg}$ enrofloxacin every $24 \mathrm{~h}$ was also initiated. Two days after admission, the ferret's general conditions improved: hyperglycemia was acceptable $(<250 \mathrm{mg} / \mathrm{dL})$, ketonuria was absent, and ketonemia was decreased $(0.7 \mathrm{mmol} / \mathrm{L})$. Mild metabolic acidosis was still present. Regular insulin therapy was suspended and subcutaneous veterinary lente insulin administration (1 IU every $12 \mathrm{~h}$ ) was initiated. Subsequently, blood gas parameters normalized, ketonemia was negative, and glycemic fluctuations were between 180-260 mg/dL. The ferret was discharged with lente insulin- and enrofloxacin-based therapy, but few days later, the symptoms relapsed with hyperthermia $\left(39.7^{\circ} \mathrm{C}\right)$, depression, anorexia, ataxia, hyperglycemia, ketonemia, and severe metabolic acidosis. The owner decided to euthanize the ferret, and necropsy immediately followed. Histological examination revealed that the left adrenal gland was composed of atypical cells of the adrenal cortical tissue compatible with carcinoma of the adrenal cortex. The right adrenal gland showed cortical hyperplasia. The pancreatic tissue revealed focal lymphoplasmacytic inflammation. The number of $\beta$ cells decreased compared with that in normal ferrets and appeared scattered. Immunoreactivity was negative for insulin. The histological features were consistent with an active chronic pancreatitis and type $1 \mathrm{DM}$ was hypothesized.

\section{Discussion}

According to its pathogenesis, DM is classified as either type 1 or type 2 (Feldman and Nelson 2004). Type 1 is characterized by pancreatic $\beta$-cells destruction, leading to absolute insulin deficiency. Although the cause of $\beta$-cells destruction in diabetic dogs is often unknown, there is evidence that immune-mediated processes do occur. Evidence of acute or chronic pancreatitis occurs in approximately $40 \%$ of diabetic dogs (Feldman and Nelson 2004). It is possible that pancreatitis may play some role in the development of immunemediated destruction of $\beta$-cells in susceptible dogs, or that the diabetic condition may be a 
risk factor for pancreatitis in this species (Fleeman and Rand 2001). By contrast, type 2 patients usually have peripheral insulin resistance, but may also concurrently have altered insulin secretion caused by $\beta$-cells dysfunction. Obesity, diet, stress, and diabetogenic drugs (i.e., steroids) may be associated with the development of type 2 DM (Feldman and Nelson 2004).

$\mathrm{DM}$ is an uncommon condition and is not well documented in ferrets. Histological examination of pancreatic tissue from diabetic ferrets is available for only two cases, where histological stains demonstrated an adequate number of $\beta$-granules in the pancreatic islets, suggesting that DM was a result of either inadequate release of insulin or peripheral insulin resistance (Carpenter and Novilla 1977; Benoit-Biancamano et al. 2005). Hillyer reported the treatment of four ferrets for persistent hyperglycemia. All four ferrets died, but histological findings were not included in the study (Hillyer 1992). Most cases of hyperglycemia in ferrets are usually transient, mostly as a postsurgical complication after resection of an insulinoma (Rosenthal 2000). In our case, histopathologic findings on the pancreatic tissue were consistent with type $1 \mathrm{DM}$. To the best of our knowledge, there are no scientific reports of type $1 \mathrm{DM}$ in ferrets. Furthermore, the concomitant presence of active chronic pancreatitis was a remarkable result. Pancreatitis is included in the list of possible underlying or concomitant diseases in diabetic patients; however, no comprehensive data on this condition is available in ferrets (Oglesbee 2006). Pancreatitis may be considered a consequence as well as a cause of DM. Patients already suffering from coexisting disorders, such as pancreatitis, renal disease, or infections, are also at greater potential risk to develop DKA. Pancreatitis and HAC have been indicated as possible causes of insulin resistance in dogs and cats (Feldman and Nelson 2004). In our case, therefore, it is possible that HAC and the presence of pancreatitis have enhanced the progression of DM to a state of DKA. In the ferret we examined, an initial diagnosis of DM and DKA as well as blood glucose and ketone monitoring were carried out using a PBGM. Such a device has not been validated for use in ferrets. Although a PBGM validation study needs to be carried out in this species, we observed a positive relationship between the blood glucose data obtained via the two methods (biochemical and PBGM), ketonemia, and ketonuria. The type of insulin normally used in ferrets is NPH (Quesenberry and Rosenthal 2003). In our case, veterinary porcine lente insulin was used with satisfactory results. This is the first case report of type $1 \mathrm{DM}$ associated with $\mathrm{HAC}$ and chronic pancreatitis in ferrets.

\section{References}

Benoit-Biancamano MO, Morin M, Langlois I. (2005) Histopathologic lesions of diabetes mellitus in a domestic ferret. Can Vet J 46: 895-897.

Carpenter JW, Novilla MN (1977) Diabetes mellitus in a black-footed ferret. J Am Vet Med Assoc 171: 890-893

Chen S (2008) Pancreatic Endocrinopathies in Ferrets. Vet Clin North Am Exot Anim Pract 11: 107-123

Feldman EC, Nelson RW (2004) Canine Diabetes Mellitus. In: Feldman EC, Nelson RW (eds) Canine and Feline Endocrinology and Reproduction. Saunders, St. Louis, pp 486-538

Fleeman LM, Rand JS (2001) Management of canine diabetes. Vet Clin North Am 31: 855-880

Hillyer EV (1992) Ferret Endocrinology. In: Kirk RW, Bonagura JD (eds) Current Veterinary Therapy XI: Small Animal Practice. Saunders, Philadelphia, pp 1185-1188

Oglesbee BL (2006) Diabetes Mellitus. In: Oglesbee BL (ed) The 5-minute Veterinary Consult: Ferret and Rabbit. Wiley-Blackwell, Hoboken, pp 32-34

Quesenberry KE, Rosenthal KL (2003) Endocrine diseases. In: Quesenberry KE, Carpenter JW (eds) Ferrets, Rabbits, and Rodents: Clinical Medicine and Surgery. Saunders, St. Louis, pp 79-90

Rosenthal KE (2000) Ferret and Rabbit Endocrine Disease Diagnosis. In: Fudge AM (ed) Laboratory Medicine: Avian and Exotic Pets. Saunders, Philadelphia, pp 319-324 